\title{
Use of computer software for technical training at rail car repair shops
}

\author{
Artem Popkov, ${ }^{1, *}$, and Sergei Bekher ${ }^{1}$ \\ ${ }^{1}$ Siberian Transport University, 630049 Novosibirsk, Russia
}

\begin{abstract}
Currently, there is a clear trend towards updating technical regulations and improving control technologies in the field of maintenance and repair of car assemblies and parts. Therefore, there is a need for software and hardware that will provide updated information to specialists of car repair shops in a timely manner. This study is aimed at creating a computer-based network training system that will increase the effectiveness of training and reliability of results. The paper describes methods for verifying and identifying training results based on an analysis of user's behavioral parameters. As a result of the research, the authors have developed a system that can verify training results with a probability of more than $95 \%$.
\end{abstract}

\section{Introduction}

In recent years, there have been repeated failures of undercarriages on railways of the Russian Federation. One of their causes are fatigue defects that have not been early detected because specialists at car repair enterprises lack professional experience and knowledge [1]. The use of network software will allow specialists to adopt training materials remotely and on-the-job, without interrupting their professional activities, and to keep themselves up to date on the latest changes in technical regulations. At present, e-learning management systems, such as Moodle, Edmodo and Schoology, are widely used. All these systems have extensive functionality, are flexible and customizable, equipped with special modules designed to manage training materials, generate statistics, etc. However, the need of fine tuning for specific demands, lack of high-quality technical support and excessive functionality make technical training systems more difficult to use by specialists of car repair shops.

In case of technical e-learning, it is impossible to guarantee the reliability of learning results [2]. Detection of attempts to falsify results or other types of fraud by trained specialists, e. g., involving a third person to pass training tests, is an acute problem.

Modern researchers mention the user's "digital handwriting" (keystroke and mouse dynamics) - unique quantitative characteristics, also called as behavioral parameters, which are determined during the user's interaction with input/output manipulators $[3,4]$. The purpose of the study is to develop a methodology that will allow car repair shops to determine the reliability of training results by analyzing students' behavioral parameters.

\footnotetext{
*Corresponding author: Zabagy@gmail.com
} 


\section{Use of computer software in training}

User's behavioral parameters were studied among specialists of car repair shops. A specialpurpose network software product was designed to conduct technical on-the-job training [5]. The most preferred way of implementing the necessary functionality is a client-server architecture [6]. A database managed by MS SQL Server database management system is used to store training materials and results. Its simplified diagram is shown in Figure 1 [7].

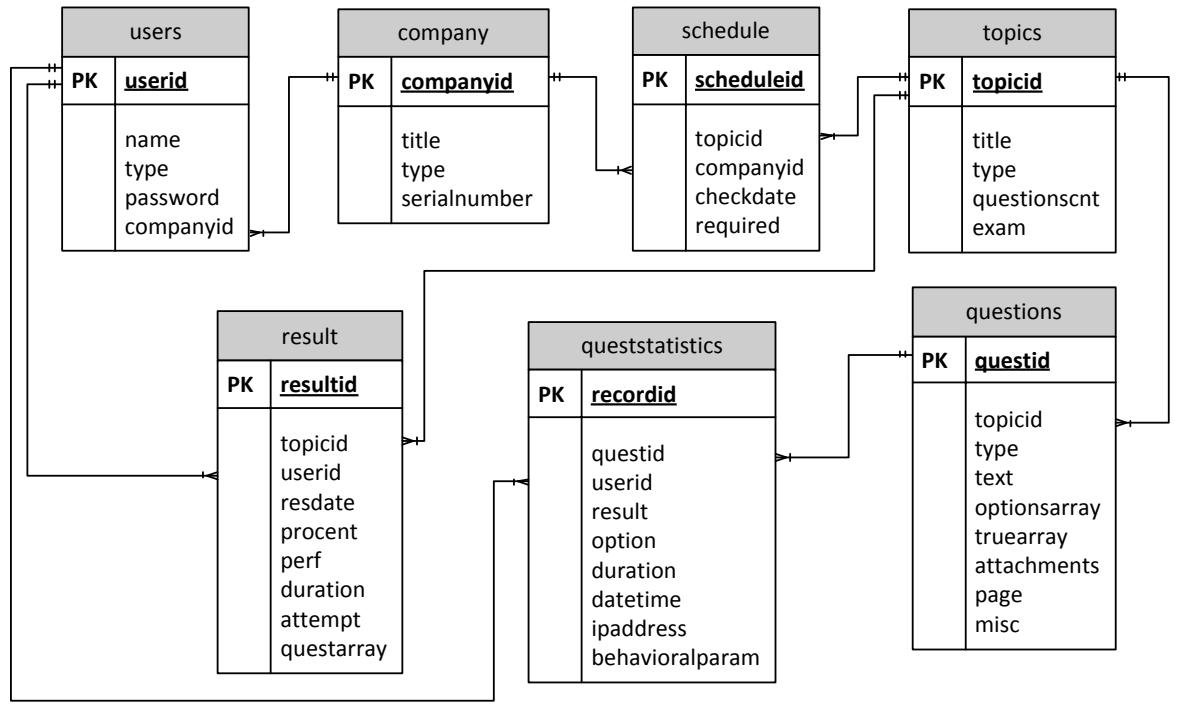

Fig. 1. Simplified diagram of the database.

Car repair shops employ non-destructive testing (NDT) specialists in various elements of the rolling stock. There are special technical regulations and training materials for these specialists [8]. Therefore, the technical training system was designed so that training materials could be hierarchically separated into several independent levels. It enables individual planning of the training process for each business unit.

Training materials include a list of topics, multimedia training aids, and a set of interactive test questions. A specialist responsible for technical training can select necessary topics according to the profile of his/her enterprise, and set key dates for them [9]. The training process has three stages: introduction to training materials, a preliminary test and a final test. When passing a preliminary test, the user has the right to use training materials and can see whether his/her answers are true or not. In the final test mode, the user will not have access to training materials and will see a final result only after completion of the test. A topic is deemed to have been passed if at least 80 percent of questions have been answered correctly both in the preliminary and final tests.

Information about progress for each topic is saved on the server. The training can be continued at any time on a PC with access to a corporate network [10]. Statistics saved in the system include the number of correct and incorrect answers, the number of views of training materials, and time spent on a question by each user.

Depending on the enterprise's profile, training can be conducted in different areas: automatic couplers, automatic brakes, general questions of NDT, bogie monitoring, railway wheel monitoring, and welding operations in repairs of undercarriages. Time spent per question varies slightly for different areas. The average value is eight seconds. It can be 
concluded that test questions for different training areas are similar in terms of difficulty (Fig. 2).

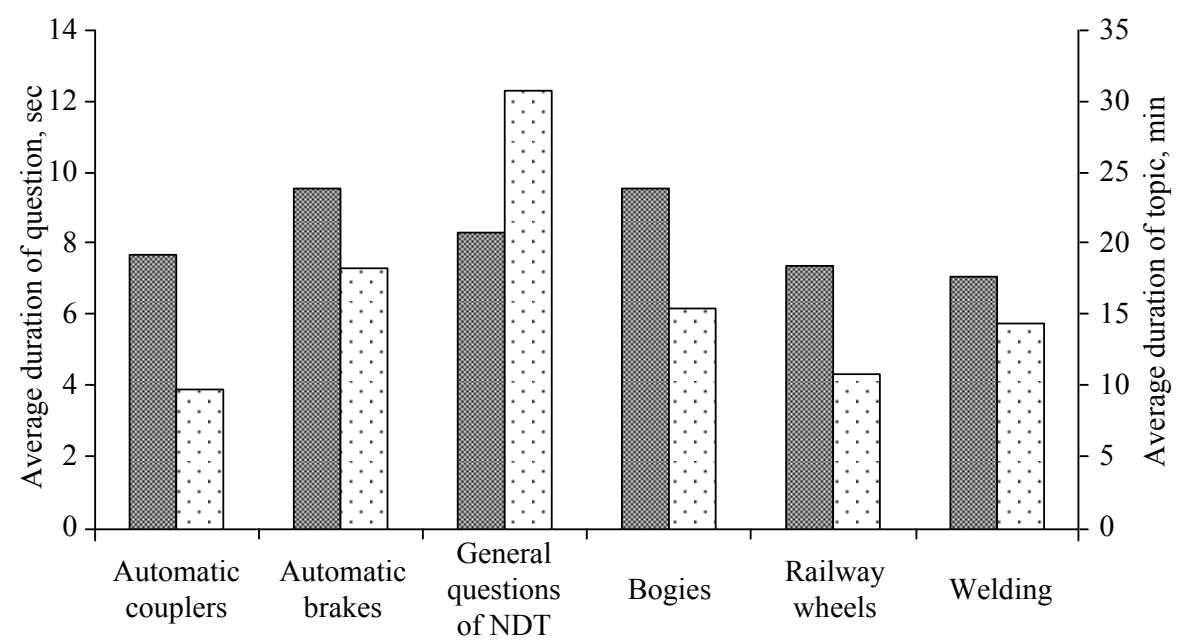

Fig. 2. Distribution of time spent per question and total time of testing per topic in different training areas.

The average time of testing for the topic "general questions of NDT" is 1.5 times or more longer than that for other training areas (Fig. 2). This is due to the fact that there are many questions in this area, used to test the knowledge of technical regulations, and users more often refer to training aids to answer them.

Although the test questions are similar in terms of difficulty, the total time of testing by different users varies. For example, user $A$ spends from three to 12 minutes to pass each topic from a set $X$. User $B$ spends seven to 18 minutes to pass each topic from the same set $X$. A relationship between times spent on each topic from the set $X$ by users A and B is close to linear (Fig. 3). The time of testing by a particular user may depend on many factors: level of attention, knowledge of the topic, etc.

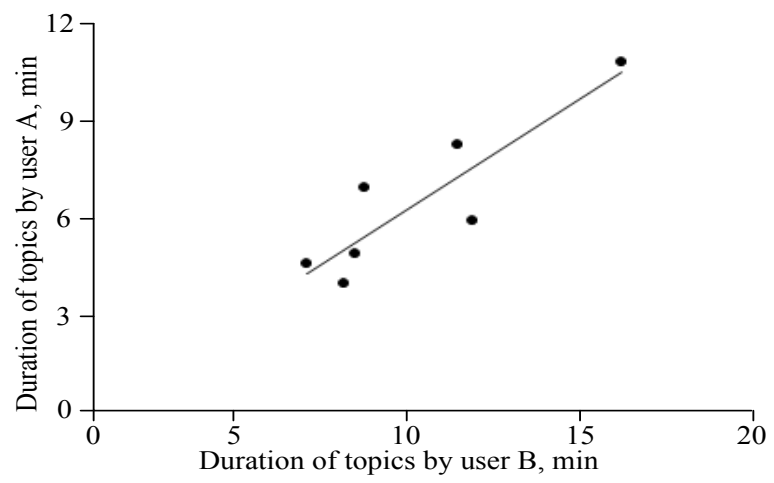

Fig. 3. A relationship between times spent on each topic by users A and B.

However, it is the number of incorrect answers that has the greatest influence on the time of testing. In most cases, a dependence of the time of testing to the number of incorrect answers is linear. 

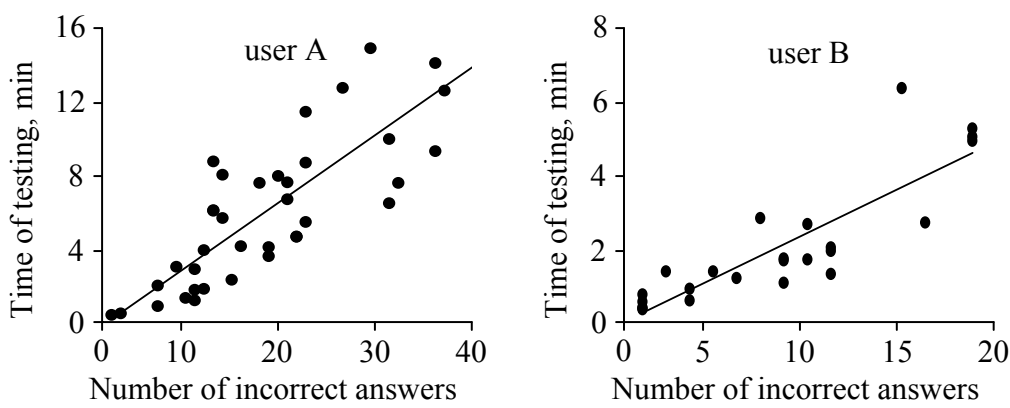

Fig. 4. Dependence of the time of testing on the number of incorrect answers.

\section{Verification of results}

The technical training system uses one-step authentication for reusable passwords. The user enters his/her unique ID (login) and reusable password. If the entered data coincides with the reference data stored on the server, authentication is considered to have been passed. This step is followed by authorization of the user and providing the access to relevant training materials and results. This access control system is sufficient for a system that processes publicly available personal data.

If the training is subject to deadlines and supervision, and the user is not personally interested in acquiring the related knowledge and skills, there is a higher probability that results will be falsified. The system cannot deny authorization to user $A$ if he/she has entered a correct login and password of user $B$. Therefore, one-step authentication with reusable passwords does not provide sufficient protection against attempts to falsify results.

When interacting with PC input/output manipulators, each user demonstrates his/her own "digital handwriting" with a variety of parameters, such as typing speed, distribution of delays between keystrokes in certain sequences, force and duration of keystrokes and mouse clicks, cursor movement speed, etc. Special-purpose input/output manipulators are required to determine the keystroke force, which makes it impossible to use these parameters in large-scale research [11].

The duration of a right mouse button click during the training process was analyzed as one of behavioral parameters. The duration of a right mouse button click for most users was found to be in the range of 80 to $1000 \mu \mathrm{s}$ [12]. The analysis of clicks for the entire training session shows that the distribution of their durations varies from user to user (Fig. 5).
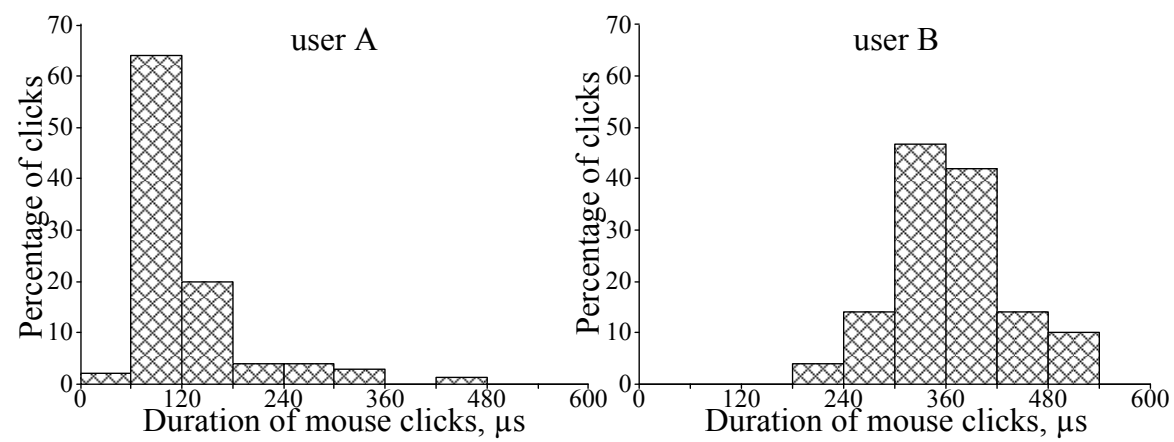

Fig. 5. Distributions of durations of mouse clicks for users A and B. 
The analysis of mouse clicks made by the users while passing a set of topics $Y$, shows that the average duration is $110 \mu$ s for user $A$ and $340 \mu$ s for user $B$, which is consistent with the previously determined distributions (Fig. 5). Standard deviations of durations of the clicks made while passing the topics from the set $V$ by users $A$ and $B$ is within $20 \mu$ s, which suggests that there is an insignificant and equal variation of experimental data for different test subjects (Fig. 6).
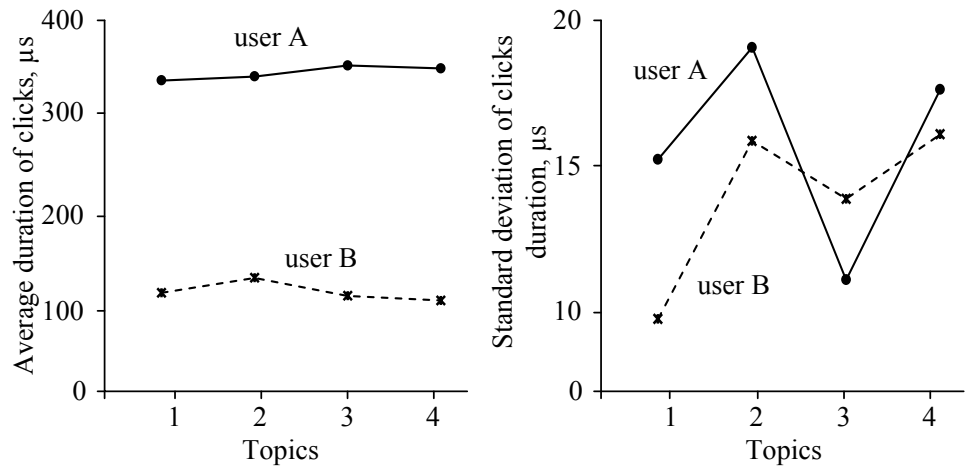

Fig. 6. Average value and standard deviation of duration of clicks in different topics for users A and B.

The duration of mouse click by a particular user $C$ is a normally distributed random variable (Fig. 5), for which the arithmetic mean $a$ and the standard deviation $\sigma$ are determined uniquely and with some accuracy. Therefore, if a value of duration is outside the range $[a-2 \sigma, a+2 \sigma]$, there is a 95 percent probability that the click does not belong to analyzed user $C$. If only one click is outside the confidence interval, it is impossible to judge whether results are falsified or not. If, however, all clicks made while passing a particular topic differ from user's typical values, results are considered to have been falsified (Fig. 7).

By analyzing training results of NDT specialists, the authors identified five of 27 available topics in which results were falsified through involving third-party users in passing a test. Throughout the entire technical training system, the number of attempts to falsify results does not exceed $1 \%$ of the total number of results.

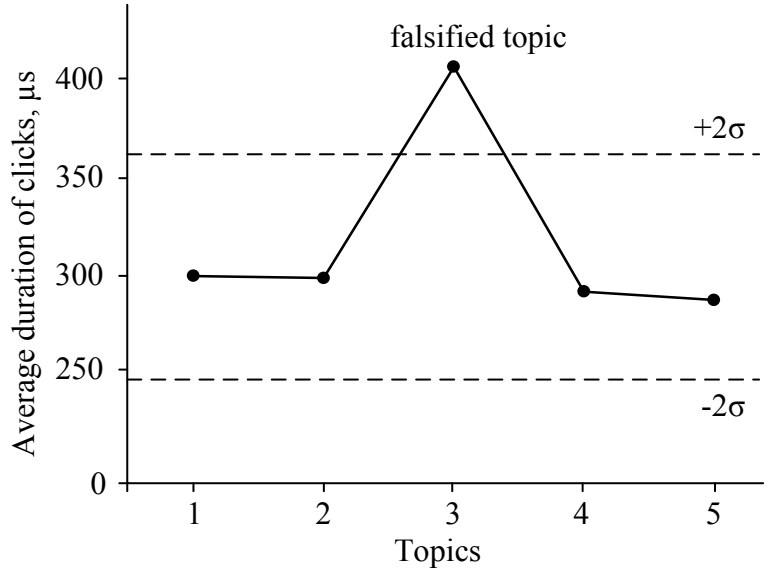

Fig. 7. Average duration of mouse clicks in different topics, including a topic with falsified results. 
In practice, it is possible that different users will have the same durations of mouse clicks. In addition, the parameters described above are influenced by a number of factors, such as user's fatigue levels, equipment, etc. Therefore, it is reasonable to use a set of several different behavioral parameters to detect falsified results more accurately.

\section{Conclusion}

Based on the results of the study, the authors proposed a system architecture to increase the efficiency of technical training by using solutions that reduce the time of training. Reducing the time of technical training will provide more time for primary activities and, therefore, increase the labor productivity of specialists at car repair shops.

The proposed technique can be used for detecting attempts to falsify training results with a probability of $95 \%$. Specialists of one of the tested enterprises falsified more than $20 \%$ of results. Throughout the entire training system, the number of attempts to falsify results does not exceed $1 \%$ of the total number of results. By expanding a range of behavioral parameters to be analyzed, it is possible to identify the user's digital handwriting (keystroke and mouse dynamics). It is expected that revealed patterns of behavioral parameters will be used to identify users and determine his/her current status.

\section{References}

1. V.S. Vorobyev, R.M. Bryzgalova, I.B. Repina, Nauka i transport [Science and transport] 3, 42-46 (2012). (in Russian)

2. S. Fuicu, M. Popa, D. Dobrilovic, M. Marcu, R. Bogdan, Brain: Broad Research in Artificial Intelligence and Neuroscience 8 (1), 52-58 (2017).

3. A. Mahfouz, T. Mahmoud, A. Eldin, Journal of Informational Security and Applications 37, 28-37 (2017).

4. M. Biasutti, Computers\&Education 12, 158-171 (2017).

5. L. Collins, X. Liang, The Turkish Online Journal of Distance Education 15, 268-281 (2014).

6. M. Adame, M. Matysek, M. Barinova, MATEC Web of Conferences 125, 02058 (2017).

7. GOST P 43.0.11-2014. Informational ensuring of equipment and operational activity. Database in technical activities (Moscow, 2015) (in Russian)

8. GOST 12.0.004-2015. Occupational safety standards system. Organization of training for safety and health at work. General requirements (Moscow, 2017). (in Russian)

9. H. Peration, Open and Distance Learning in the Developing World (Routledge, New York, 2000).

10. A.A. Popkov, S.A. Bekher, Method to testing and/or polling remote users, RU Patent 2625554 (2017).

11. S.A. Bekher, Thesis of Candidate of Sciences, Siberian Transport University, Novosibirsk, 2004 (in Russian)

12. S.A. Bekher, A.A. Popkov, The program of user identification in the process of remote testing. Registration No. 2017610808, 18.01.2017 (Moscow, 2017). (in Russian) 